\title{
Cross-Talk among Glucocorticoids, Glucocorticoid Receptors and Cytokines Pilots Inflammatory, Endocrine, Immune and Metabolic Responses in HIV Infection
}

\section{Guido Norbiato*}

Department of Endocrinology and Metabolism, L. Sacco University Hospital, Milan, Italy

\begin{abstract}
A number of diseases including viral and bacterial infections, inflammatory and auto-immune disorders is associated with glucocorticoids resistance. Tissue resistance to glucocorticoids is observed in untreated HIV infected patients with hypercortisolism. These patients show a complete inability of glucocorticoids to exert their effect, full Addisonian symptoms and an impressive increase in type-1 Th-directed cellular immunity. This clearly suggests a severe receptor resistance to glucocorticoids. Removal of glucocorticoids by adrenal insufficiency or decreased glucocorticoid receptors sensitivity, likely induced by HIV RNA, endotoxin and lipopolysaccarides, significantly enhances morbidity and mortality in such patients. Highly active antiretroviral therapy is an important advance in the treatment of HIV infection since confers survival and acceptable conditions of life, but the suppression of viral replication is not associated with reconstitution of the immune function. This allows persistence of chronic inflammatory disease and activation of local $11 \beta$ hydroxy steroid dehydrogenase type- 1 and type- 2 with associated increases in glucocorticoids and mineralcorticoids production. Glucocorticoids and their receptors have a central role in the control of innate and adaptive immune responses. In turn, cytokines signaling pathway may influence neuroendocrine function through changes in the receptors sensitivity and function. Evidence shows that glucocorticoids system works in concert with other systems, including the immune, metabolic and renin-angiotensin aldosterone systems. Imbalance of this network mainly drives to chronic inflammation and the metabolic syndrome with its component of insulin resistance, dyslipidemia and cardiovascular complications. Therapeutic strategies to restore the integrity of glucocorticoid receptor function and reconstitute the immune function are needed to keep HIV infected patients away from complications. Current therapeutic approaches tend to inhibit the $11 \beta$ hydroxy steroid dehydrogenase type- 1 activity and the renin-angiotensin-aldosterone system function. Both these treatments may reduce inflammation.
\end{abstract}

Keywords: Glucocorticoids; Glucocorticoid receptors; Glucocorticoid receptor resistance; Insulin resistance; Metabolic syndrome; $\mathrm{Cy}$ tokines; $11 \beta$ hydroxy steroid dehydrogenase type-1 and type-2; Reninangiotensin-aldosterone system; Endothelium

Abbreviations: FFA - Free Fatty Acid; GR - Glucocorticoid Receptors; 11 beta HSD1 - 11 $\beta$ Hydroxy Steroid Dehydrogenase Type1; HSD2 - 11 $\beta$ Hydroxy Steroid Dehydrogenase Type-2; HAART - High Active Antiretroviral Therapy; HPA axis - Hypothalamus-PituitaryAdrenal axis; IL-1 - Interleukine-1; IFNa - Interferon alfa; IFN $\gamma$ Interferon Gamma; Il-6 - Interleukin-6; LPS - Lipopolysaccarides; MR - Mineralcorticoid Receptor; RAAS - Renin-Angiotensin-Aldosterone System; TNFa - Tumor Necrosis Factor Alfa; Th-1 - Thelper-1; Th-2 Thelper-2

\section{Introduction}

Resistance to the anti-inflammatory and immune effects of glucocorticoids is one of the most important clinical aspects of HIV infection. Since the responsiveness of the target tissues to glucocorticoids is crucial for the end effect of this hormone and any generalized changes in the glucocorticoid signaling would be expected to be followed by compensatory changes in the glucocorticoid regulatory mechanism, this will result in a new adaptive equilibrium in the hormonal, immune and metabolic system function. Glucocorticoids play an essential role in restraining inflammatory, neuro-endocrine and immune responses to a variety of challenges including pathogens exposure and stress [1]. It is now recognized that changes in Glucocorticoid Receptor (GR) sensitivity have a role in the pathogenesis of a number of disorders including auto immune and inflammatory diseases which are believed to be related both to impaired or increased functioning of GR.
During human immune deficiency HIV virus infection, activated immune cells such as lypogenic pro-inflammatory cytokines interleukine -1 (IL-1), interferon alfa ( IFNa), interferon gamma ( IFN $\gamma$ ), tumor necrosis factor alfa (TNF $\alpha)$ stimulate the release of systemic glucocorticoids which provide important control over the immune system [2]. AIDS patients present several manifestations compatible with an excessive production of cortisol. A decreased sensitivity to glucocorticoids has been shown in the immune cells of HIV infected individuals with high circulating levels of cortisol. Competitive binding studies performed on mononuclear lymphocytes taken from these patients revealed a low receptor affinity for glucocorticoid while the number of receptors was increased. These changes were associated to an impressive increase in type-1 Th-directed cellular immune response no more suppressed by high levels of circulating cortisol, giving evidence that HIV infected patients have become resistant to cortisol [3]. Interestingly these receptor changes were similar to those induced by endotoxin- lipopolysaccarides (LPS) which have been shown to

*Corresponding author: Emeritus Professor Guido Norbiato, Via V. Dandolo, 27 -21100 Varese - Italy, E-mail: guidonorbiato@libero.it

Received February 23, 2013; Accepted March 15, 2013; Published March 24 2013

Citation: Norbiato G (2013) Cross-Talk among Glucocorticoids, Glucocorticoid Receptors and Cytokines Pilots Inflammatory, Endocrine, Immune and Metabolic Responses in HIV Infection. J AIDS Clinic Res S5: 007. doi:10.4172/2155-6113.S5-007

Copyright: ( $) 2013$ Norbiato G. This is an open-access article distributed under the terms of the Creative Commons Attribution License, which permits unrestricted use, distribution, and reproduction in any medium, provided the original author and source are credited. 
increase GR numbers but decrease affinity [4]. Treatment of HIV infection with High Active Antiretroviral Therapy (HAART) confers survival and quality of life benefit. However these significant benefits are at the cost of metabolic complications and risk of type-2 diabetes and cardiovascular disease. These chronic diseases add complexity to the standards of the care in HIV infection and much remains unknown about the natural histories of the metabolic syndrome, diabetes, cardiovascular complications and their therapeutic approaches. This review will focus on the potential contribution of inflammation and activation of the cytokine signaling pathway to GR resistance and its relevance to major complications of HIV infection.

\section{Glucocorticoids and GR: A Functional Unit}

GR is essential to life and the GR anti-inflammatory action may be most critical for survival. It is important to remember that GR is ubiquitous in the human body and that, upon cortisol binding to GR is activated, this steroid regulates the transcription expression of the target genes and downstream physiological function. Glucocorticoids exert many effects through cytosolic receptors, member of the nuclear hormone receptor super family, which also includes the thyroid hormone, mineral corticoid, estrogen and progesterone receptors. Upon ligand activation, GR is released from the protein complex, dimerizes and translocates in the nucleus where it binds to specific DNA sequences called glucocorticoid response elements (GRE). Thus, GR function has a ligand dependent transcription factor [5]. GR activation by glucocorticoids primarily represses gene transcription by interfering with the action of signaling pathways such as nuclear factor $\mathrm{KB}\left(\mathrm{NF}_{\mathrm{k}} \mathrm{B}\right)$ and activator protein (AP-1). It is through this mechanism that glucocorticoids exert their anti-inflammatory action [6]. Cortisol participates to physic and psychic responses to stress, inflammation and immune challenges. Circulating cortisol is produced by biosynthesis in the adrenal glands, regulated by the HPA axis, and by activation of hydroxysteroid dehydrogenase type 1 enzyme (11ßHSD1) which catalyzes the conversion of inactive cortisone into active cortisol, mainly in adipose, liver, muscle and brain tissues [7].

Another enzyme, the $11 \beta$ hydroxysteroid dehydrogenase type 2 $(11 \beta \mathrm{HSD}-2)$, participates to the process, catalyzing the conversion of active cortisol into inactive cortisone in target tissues such as kidney, heart, endothelium and colon. These enzymes also interact with the mineral corticoid receptor (MR) and directly regulate aldosterone production [8]. Thus the receptor responsiveness to glucocorticoid is crucial for the responses to HPA axis, 11ßHSD-1, 11ßHSD-2 and MR.

\section{Endocrine and Immune Alterations in Acute HIV Infection}

A certain number of untreated HIV infected individual's present hypercortisolim and an acquired form of glucocorticoid resistance. This may be related to a chronic activation of HPA axis by inflammatory cytokines. Infact TNFa, IL-1 and IL-6 may account for most of HPA axis-stimulating activity [9]. IL-6 stimulates both ACTH and cortisol in men [10] and glucocorticoid suppresses IL- 6 production by decreasing the transcription rates of the gene for this interleukin [11]. However, chronically elevated levels of inflammatory cytokines may restrain HPA axis by blocking the stimulatory effect of ACTH and CRH on the pituitary and adrenal cortex [12]. Thus we are faced with a complex mechanism aimed to regulate glucocorticoids function. An important question regards the role that glucocorticoids and their receptors have in HIV disease progression in untreated HIV infected individuals. In the advanced stage of the disease these patients may present signs and symptoms of adrenal insufficiency such as skin hyperpigmentation, chronic fatigue, hypotension but high cortisol levels in the plasma. Progression of the disease in such patients is also associated with a shift of the cytokine pattern from a Th-1 to a Th-2 type, which allows replication to the virus to proceed [13]. Association of hypercortisolism with addisoniam symptoms and the impressive increase of T Helper-1 (Th-1) directed cellular immunity strongly suggest that HIV infected patients become resistant to glucocorticoids [3]. A moderate resistance to glucocorticoid has been detected in more than half of untreated HIV infected patients in the early phase of the disease [14] which suggests that HIV retrovirus infection induces progressive changes in receptors function and that these changes may have the effect of glucocorticoid antagonists in reversing the cytokine pattern from the Th-2 to Th-1 sub-type.

In HIV infected patients, intact HPA axis and glucocorticoid response are needed for survival to viral and bacterial infection. GR is a target for toxins. Endotoxemia is associated with altered innate and adaptive immune response in untreated HIV patients [15]. An excess of TNFa is considered the most likely cause of enhanced LPS/ endotoxin induced mortality. Removal of endogenous glucocorticoids by adrenal insufficiency or decreased GR sensitivity significantly enhances the mortality from endotoxin, LPS and viral HIV RNA [4]. When invading pathogens are not controlled as occurs in HIV retrovirus infection, the immune system starts destroying immune cells, and consequently causes auto-immune and inflammatory diseases. Continuous stimulation of T-cells also occurs leading to the functional exhaustion of effector T-cells. During chronic HIV retrovirus infection several inhibitory molecules are over expressed on virus specific CD-4 and CD-8 T-cells and this is associated with functional exhaustion leading ultimately to delation of the exhausted T-cells. IL-2 production and T-cell proliferation potentially are lost first, TNFa production and cytotoxic capacity disappears later; finally exhausted cells are deleted via apoptosis $[16,17]$. Increments of glucocorticoids production may be also responsible for functional exhaustion of T-cells and increased apoptosis. GR resistance may counteract these effects.

\section{Chronic Inflammatory Disease in HAART Treated HIV Patients}

Investigation of the HPA axis in chronic inflammation has shown an anti-inflammatory influence of the HPA axis, equivocal results with respect to the levels of adrenocortical hormone ACTH and cortisol, inadequate low secretions of adrenal hormone in relation to inflammation, modulating role of a TNFa and IL-6 on the HPA axis and GR hyposensitivity. Basis of these alterations is an adaptive program selected for short lived inflammatory responses which becomes a disease inherent pathologic factor. If this adaptive program continues longtime, it can drive systemic disease sequel such chronic inflammatory disease and the metabolic syndrome [18]. The metabolic syndrome refers to unbalances of the endocrine, immune and metabolic functions which lead to the development of obesity, primarily of the visceral type, with its components of insulin resistance, dyslipidemia, hyperglycemia, endothelium dysfunction, blood hypercoagulation and hypertension. The risk of developing the metabolic syndrome seems related to HIV infection, specific antiretroviral agents, inflammation and body fat distribution. The prevalence of metabolic syndrome in AIDS ranges from 17 to 34 percent and is partly dependent on the diagnostic definition used [19]. Seen the slow evolution of the disease an increased prevalence of metabolic and cardiovascular complications can be expected.

Although circulating cortisol levels are normal in HIV patients 
treated with HAART, the $11 \beta$ HSD-1 enzyme is activated. Overexpression of $11 \beta$ HSD-1, induced by the inflammatory cytokine IL-1 and TNF $\alpha$ - is associated with increased production of intracellular cortisol in target tissues [7]. $11 \beta$ HSD-1 is also elevated during hyperglycemia, the effect being mediated by an increase in cytosolic exose-6-phosphate dehydrogenase, which in turn drives $11 \beta$ HSD1 activity and local glucocorticoid generation [20]. Interestingly glucocorticoids are powerful inhibitors of IL- $1 \beta$ and TNFa secretion and of many of the inflammatory effects of these cytokines [21], and at the same time they can limit inflammation by inducing the reductase activity of $11 \beta$ HSD-1. These processes occur in different times thus, dual actions of $11 \beta$ HSD-1 and TNFa, may balance the inflammatory effect and contribute to the ultimately necessary arrest of inflammation or its persistence. Dual actions of $11 \beta$ HSD-1 and $11 \beta$ HSD-2 which control access of cortisol and cortisone to GR and MR, determine the anti-inflammatory effect of the glucocorticoid or the pro-inflammatory effect of aldosterone [22]. Since pro-inflammatory cytokines reduce GR sensitivity, this must be considered an important mechanism leading to persistence of inflammation in HIV infected patients.

\section{Metabolic and Cardiovascular Complications}

Visceral obesity is one major risk factor for cardiovascular and metabolic disease. Studies have shown that T-cells are key regulators not only of adipose inflammation but also of systemic glucocorticoids and insulin activity. Adipose tissue inflammation in the metabolic syndrome, results in activation of the $11 \beta$ HSD-1, cortisol resistance, insulin resistance and a shift from expansion of subcutaneous fat to deposition of abdominal and ectopic fat. Protease inhibitors- associated syndrome of peripheral lipodystrophy, hyperlipidemia and insulin resistance has been described. This effect of protease inhibitors may be directed or mediated by inflammation. Glucocorticoids are recognized to play a key role in the pathogenesis of insulin resistance in HAART treated patients. As cortisol acts as natural antagonist of insulin and has the same target tissues of insulin, changes in the sensitivity of the glucocorticoid and insulin receptors may be a fundamental mechanism by which these hormones regulate lipid and glucose metabolism in tissues such as fat, liver, muscle and brain. With insulin resistance, skeletal muscle, liver and adipose tissue metabolism is altered. Decreased glucose metabolism is often correlated with elevation in FFA metabolism; glucocorticoid- induced insulin resistance is associated with augmented FFA utilization [23]. Specific inactivation of hepatic GR reduces elevated glucose output and improves hyperglycemia. These metabolic alterations are widely believed to predispose to diabetes mellitus type- 2 and cardiovascular complications.

Endothelial dysfunction is an important event in the initiation and progression of atherosclerotic-vascular diseases associated with HIV infection. Endothelium activation leads to a pro-inflammatory proliferative and protrombotic state of the endothelium [24]. The presence of the insulin receptors on endothelial cells is well documented and endothelium is now emerged as a potential important target tissue for insulin. Diabetes and inflammation contribute to atherosclerosis and associated endothelium damaged by promoting the position of modified lipids in the endothelium and by inhibiting nitric oxide production [23]. Since glucocorticoids produce whole body insulin resistance changes in glucorticoid production and/or glucocorticoid receptor function may participate to endothelium dysfunction in HIV infected individuals. However experiments in rat have recently shown that endothelial cells may be resistant to glucocorticoids, so explaining the inefficiency of glucocorticoids in the treatment of vasculitis [25]. Interestingly $11 \beta$ HSD-2 enzyme is mainly expressed in vascular endothelium and heart where induces activation of $\mathrm{MR}_{\mathrm{s}}$ and increases the production of mineralcorticoid which are pro-inflammatory hormones.

In the heart, elevated FFA has been implicated in metabolic, morphological and mechanical changes and lypotoxicity [23]. During metabolic stress, such as diabetes and insulin resistance characterized by inadequate glucose utilization, cardiac FFA consumption supersedes glucose oxidation. Thus, increased FFA delivery following glucocorticoids, alters FFA composition in the heart. This may be an important mechanism by which heart is damaged.

Finally, very early increase of blood pressure may be responsible for pathologic changes in the endothelium and heart due to activation of the RAAS. Blockade of this system improves the release of pancreatic insulin, increases insulin-mediated glucose utilization as well as reduces progression of inflammation and cardio metabolic disease [22].

\section{Discussion}

An intact HPA axis and preserved glucocorticoid receptor sensitivity is needed in HIV patients for survival to viral and bacterial infection during HIV disease. Activated immune cells, secrete pro-inflammatory cytokines that stimulate the release of cortisol which, in turn, provides important controls of the immune system and inflammation. This does not occur in acutely infected HIV patients as their GR, target for HIVRNA, endotoxin and LPS [4], become resistant to cortisol. Cortisol resistance also occurs in HAART treated patients as invading pathogens are not contained by adequate immune-endocrine response. This results in a chronic inflammatory disease. Persistence of inflammation in these patients triggers the cortisone-cortisol shuttle resulting in an increased intra-cellular cortisol production. The consequence is that plasma cortisol concentration remains within normality, but 11 $\beta$ HSD1 activity and cortisol production increase in macrophages, adipose tissue, liver, muscles and brain, until the inflammation is resolved. The immune system controls glucocorticoids and their receptors. Infact a number of cytokines including interleukin IL-1, IL-2, TNFa and INF $\alpha$ influence the neuro-endocrine and GR function [26]. In turn, the cytokines system is under the control of glucocorticoids hormones and their receptors. Thus we are faced with a complex network which interacts and works in concert with other systems, particularly the metabolic and the RAAS system.

Since cortisol acts as a natural antagonist of insulin by reducing insulin sensitivity in the same target tissues, changes in receptors sensitivity to both cortisol and insulin may be considered a fundamental mechanism by which cortisol and insulin lead to metabolic syndrome and cardiovascular complications. Focus of this review is also the endothelium dysfunction that has a key role in the initiation and progression of atherosclerotic and vascular complications [24].

\section{Conclusion}

Evidence from studies in HIV infected patients shows that glucocorticoids and their receptors play an essential role in the regulation of endocrine, immune, metabolic and inflammatory responses to a variety of challenges including pathogens exposure inflammation and stress. Glucocorticoids and their receptors are the basis of an adaptive program for short lived inflammatory response, but if inflammation persists, as it occurs in HAART treated patients, a new adaptive equilibrium between cortisol and its receptors can be reached, to limit metabolic and vascular complications. Continuous resetting of the glucocorticoid receptors during chronic inflammation may explain the low increasing morbidity and the longtime occurring before severe complications become manifest. 
Citation: Norbiato G (2013) Cross-Talk among Glucocorticoids, Glucocorticoid Receptors and Cytokines Pilots Inflammatory, Endocrine, Immune and Metabolic Responses in HIV Infection. J AIDS Clinic Res S5: 007. doi:10.4172/2155-6113.S5-007

Future therapeutic strategies will be designed to reconstitute the immune function, restrain inflammation and regulate glucocorticoidsglucocorticoid receptors cross-talk. Current treatments are based on $11 \beta$ HSD-1 antagonists, which are expected to reduce cortisol production and prevent insulin resistance, and angiotensin1-aldosterone blockers or renin inhibitors that may be effective in preventing and treating hypertension, atherosclerosis and metabolic complications.

\section{References}

1. Raison CL, Miller AH (2003) When not enough is too much: the role of insufficient glucocorticoid signaling in the pathophysiology of stress-related disorders. Am J Psychiatry 160: 1554-1565.

2. Coutinho AE, Chapman KE (2011) The anti-inflammatory and immunosuppressive effects of glucocorticoids, recent developments and mechanistic insights. Mol Cell Endocrinol 335: 2-13.

3. Norbiato G, Bevilacqua M, Vago T, Baldi G, Chebat E, et al. (1992) Cortisol resistance in acquired immunodeficiency syndrome. J Clin Endocrinol Metab 74: 608-613.

4. Marketon JI, Sternberg EM (2010) The glucocorticoid receptor: a revisited target for toxins. Toxins (Basel) 2: 1357-1380.

5. Kassel O, Herrlich P (2007) Crosstalk between the glucocorticoid receptor and other transcription factors: molecular aspects. Mol Cell Endocrinol 275: 13-29.

6. Aranda A, Pascual A (2001) Nuclear hormone receptors and gene expression. Physiol Rev 81: 1269-1304

7. Tomlinson JW, Moore J, Cooper MS, Bujalska I, Shahmanesh M, et al. (2001) Regulation of expression of $11 \beta$-hydroxysteroid dehydrogenase type- 1 in adipose tissue: tissue-specific induction by cytokines. Endocrinology 142: 1982-1989

8. Odermatt A, Arnold P, Frey FJ (2001) The intracellular localization of the mineralocorticoid receptor is regulated by 11 beta-hydroxysteroid dehydrogenase type 2. J Biol Chem 276: 28484-28492.

9. Norbiato G, Bevilacqua M, Vago T, Clerici M (1996) Glucocorticoids and interferon-alpha in the acquired immunodeficiency syndrome. J Clin Endocrinol Metab 81: 2601-2606.

10. Mastorakos G, Chrousos GP, Weber JS (1993) Recombinant interleukin-6 activates the hypothalamic-pituitary-adrenal axis in humans. J Clin Endocrinol Metab 77: 1690-1694.

11. Zanker B, Walz G, Wieder KJ, Strom TB (1990) Evidence that glucocorticosteroids block expression of the human interleukin- 6 gene by accessory cells. Transplantation 49: 183-185.
12. Chrousos GP (1995) The hypothalamic-pituitary-adrenal axis and immunemediated inflammation. N Engl J Med 332: 1351-1362.

13. Clerici M, Hakim FT, Venzon DJ, Blatt S, Hendrix CW, et al. (1993) Changes in interleukin-2 and interleukin-4 production in asymptomatic, human immunodeficiency virus-seropositive individuals. J Clin Invest 91: 759-765.

14. Vagnucci AH, Winkelstein A (1993) Circadian rhythm of lymphocytes and their glucocorticoid receptors in HIV-infected homosexual men. J Acquir Immune Defic Syndr 6: 1238-1247.

15. Bukh AR, Melchjorsen J, Offersen R, Jensen JM, Toft L, et al. (2011) Endotoxemia is associated with altered innate and adaptive immune responses in untreated HIV-1 infected individuals. PLoS One 6: e21275.

16. Wherry EJ, Blattman JN, Murali-Krishna K, van der Most R, Ahmed R (2003) Viral persistence alters CD8 T-cell immunodominance and tissue distribution and results in distinct stages of functional impairment. J Virol 77: 4911-4927.

17. Viganò S, Perreau M, Pantaleo G, Harari A (2012) Positive and negative regulation of cellular immune responses in physiologic conditions and diseases. Clin Dev Immunol 2012: 485781.

18. Straub RH, Cutolo M, Fleck M (2011) Rheumatoid arthritis recapitulates events relevant in blastocyst implantation and embryogenesis: a pathogenetic theory. Semin Arthritis Rheum 41: 382-392.

19. Lakka HM, Laaksonen DE, Lakka TA, Niskanen LK, Kumpusalo E, et al. (2002) The metabolic syndrome and total and cardiovascular disease mortality in middle-aged men. JAMA 288: 2709-2716.

20. Chapman KE, Coutinho A, Gray M, Gilmour JS, Savill JS, et al. (2006) Loca amplification of glucocorticoids by 11 beta-hydroxysteroid dehydrogenase type 1 and its role in the inflammatory response. Ann N Y Acad Sci 1088: 265-273.

21. Escher G, Galli I, Vishwanath BS, Frey BM, Frey FJ (1997) Tumor necrosis factor alpha and interleukin 1 beta enhance the cortisone/cortisol shuttle. J Exp Med 186: 189-198.

22. Norbiato G (2012) Endocrine, metabolic, and immunologic components of HIV infection. Ann N Y Acad Sci 1262: 51-55.

23. Qi D, Rodrigues B (2007) Glucocorticoids produce whole body insulin resistance with changes in cardiac metabolism. Am J Physiol Endocrinol Metab 292: E654-E667.

24. Hürlimann D, Weber R, Enseleit F, Lüscher TF (2005) [HIV infection, antiretroviral therapy, and endothelium]. Herz 30: 472-480.

25. Koenen P, Barczyk K, Wolf M, Roth J, Viemann D (2012) Endothelial cells present an innate resistance to glucocorticoid treatment: implications for therapy of primary vasculitis. Ann Rheum Dis 71: 729-736.

26. Pace TW, Hu F, Miller AH (2007) Cytokine-effects on glucocorticoid recepto function: relevance to glucocorticoid resistance and the pathophysiology and treatment of major depression. Brain Behav Immun 21: 9-19. 